\title{
Allogenous Bone Grafts Improved by Bone Marrow Stem Cells and Platelet Growth Factors: Clinical Case Reports
}

\author{
*Humberto Filho Cerruti, †Irina Kerkis, $\ddagger$ Alexandre Kerkis, \\ $\S$ Nelson Hidekazu Tatsui, $†$ Adriana da Costa Neves, IIDaniela Franco Bueno, \\ and **Marcelo Cavenaghi Pereira da Silva
}

\begin{abstract}
*Clínica CERA LTDA; †Laboratório de Genética, Instituto Butantan; \$Genética Aplicada Atividades Veterinárias LTDA; $\S$ Hospital das Clínicas da Faculdade da Medicina da Universidade de São Paulo; IlCentro de Estudos do Genoma Humano, Instituto de Biociências da Universidade de São Paulo; and **Departamento de Morfologia e Genética, Universidade Federal de São Paulo-Escola Paulista de Medicina, São Paulo, Brazil
\end{abstract}

\begin{abstract}
In order to increase the amount of available bone where dental implants must be placed, the present study has associated platelet-rich plasma (PRP) and mononuclear cells (MNCs) from bone marrow aspirate and bone scaffold (BS) in 32 patients aged between 45 and 75 years old. The MNC attainment and the adherence to the BS were confirmed through histology, cell culture, and scanning electron microscopy. The clinical results, analyzed by
\end{abstract}

computed tomography, have showed that the scaffolds were well integrated and adapted to the cortical bone. We can conclude that the process of healing observed in the patients was due to the presence of mesenchymal stem cell in MNC fraction in the bone grafts. Key Words: Alveolar ridge augmentation-Blood platelets-Bone marrow cells-Dental implantation-Stem cell.
Dental implants in aging patients are expected to increase in a disproportionate number. People are living longer and the problem with the lack of teeth continues to be more relevant among the elderly than in other age groups (1). However, there are several problems that surgeons must be aware of, such as the localization of hypermineralized osteopetrosis and areas of osteopenia, which are characterized by an increasing cortical porosity and a decreasing density of cortical bone $(1,2)$. Besides, the amount of bone in the surgical area, in height and in width, due to atrophy of the maxilla could determine the success of the implant (1).

To overcome this problem, different therapies had been developed, such as the use of bone grafts (allografts, xenografts, alloplasts, and autografts) and techniques that use apheresis products like platelet-

doi:10.1111/j.1525-1594.2007.00374.x

Received January 2007.

Address correspondence and reprint requests to Dr. Marcelo Cavenaghi Pereira da Silva, Departamento de Morfologia e Genética, Universidade Federal de São Paulo, Rua Botucatu 740, 04023-900, São Paulo, Brazil. E-mail: marcaven@gmail.com rich plasma (PRP) and platelet-poor plasma (PPP) alone or in combination with stem cells $(3,4)$, to provide regeneration of local bone and better implant osseointegration (5).

There are two principal lineages of stem cells in mononuclear fraction of the bone marrow aspirate (BMA): one responsible for hematopoiesis and another called bone marrow stromal cells regarded as mesenchymal stem cell (MSC) population (6). The role of MSC in repairing tissues has been studied for decades $(6,7)$. These cells have been shown to differentiate in condroblasts (8), myoblasts (9), adipocytes (10), nerve-like cells (11), and osteoblasts (12), and when combined with mineralized three-dimensional (3D) scaffolds they can form a primary bone tissue that is highly vascularized (13).

The healing process is preceded by repair and regeneration, which involves many cell types and the addition of growth factors. Previously, an experimental canine animal model showed that MSC and PRP significantly improved the bone density when applied together with dental implant (3). Furthermore, it was also reported that in vitro cultivated MSC isolated from iliac crest, used together with PRP, can reduce 
probing depth and improve attachment levels in human periodontal disease (14).

The aims of our work are to describe a tissue regeneration technique using allogenous bone obtained from trustworthy osseous banks in combination with autologous mononuclear cells (MNCs) from BMA isolated from aging patients' iliac or sternum and growth factors (PRP), and also to demonstrate its clinical results in short and long terms.

\section{MATERIAL AND METHODS}

All the procedures were performed according to the Institutional Ethics Committee requirements and with formal consent given by each patient.

- PPP and PRP extraction: Apheresis (Haemonetics, Braintree, MA, USA) of the peripheral blood from the patients was performed to obtain PRP and PPP.

Whole blood samples of the patient were used for PPP extraction. Ten milliliters of PPP was placed in a tube containing borosilicate spheres, $66 \%$ ethanol, and $25 \mathrm{mM} \mathrm{CaCl}_{2}$ (per Gail Rock). The tube was agitated and the mixture was left to coagulate. After coagulation, the tube was vigorously agitated and autologous thrombin was obtained in the supernatant.

The remaining PPP was used to produce fibronectin and laminin, after being frozen at $-85^{\circ} \mathrm{C}$ to obtain a cryoprecipitate. At the same time, under laminar flow, the scaffold of iliac bone obtained from the Tissue Bank (Department of Orthopedics and Rehabilitation r-12, University of Miami School of Medicine, Miami, FL, USA) was adapted to the patients' surgical prototype using a drill.

The prepared scaffold was placed in a vacuum receptacle where a syringe containing fibronectin and laminin was adapted. At this time, the scaffold absorbs fibronectin and laminin which are known to attract MSC (15).
- Bone marrow aspiration procedure: A hematologist expert in medullae transplants performed puncture aspiration of $5 \mathrm{~mL}$ of bone marrow from the patients' iliac or sternum. These punctures were obtained by scraping the needle on the bone wall. Two punctures were made in order to obtain $5 \mathrm{~mL}$ of BMA.

In another vacuum receptacle, a syringe with a filter membrane of $80 \mu \mathrm{m}$ in the bottom was adapted and the scaffolds were placed over it. The BMA was poured in the syringe and at this time, the scaffolds absorbed the bone marrow cells (Fig. 1A). After this procedure, the bone scaffold (BS) with the cells from BMA was placed in the surgical area.

- In vitro cultivation of MNC fraction on BS: MNC fraction from the BMA was allowed to adhere on BS and was cultivated during $24 \mathrm{~h}$ in $35 \mathrm{~cm}^{2}$ Petri dishes (Corning, New York, NY, USA) with Dulbecco's modified Eagle's medium supplemented with $15 \%$ fetal bovine serum, 100 units $/ \mathrm{mL}$ penicillin, $100 \mu \mathrm{g} / \mathrm{mL}$ streptomycin, and $2 \mathrm{mM} \mathrm{L-}$ glutamine (all reagents from Invitrogen, Carlsbad, CA, USA). These BS were used only for electron microscopy observations.

- Antibodies and immunofluorescence analysis: BS with adhered cells, which were not used in the surgery, were washed twice in phosphate-buffered saline (PBS) solution (0.01 M, pH=7.4) and treated with $0.05 \%$ trypsin solution (Invitrogen) for $30 \mathrm{~min}$ at $37^{\circ} \mathrm{C}$. The BS was removed and cell suspension was harvested using the aforementioned culture medium. The cells were washed twice in PBS and fixed with 4\% paraformaldehyde (Sigma, St. Louis, MO, USA). Antibodies against human MSC surface antigens SH-2 (CD105, endoglin), SH-3, and SH-4 (CD73) were graciously provided by Arnold I. Caplan (Skeletal Research Center, Case Western Reserve University, Cleveland, $\mathrm{OH}, \mathrm{USA})$. The immunostaining protocol that was used is described as follows: cell suspen-
A

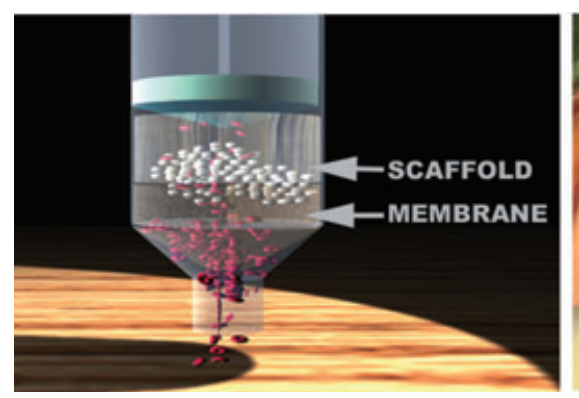

B

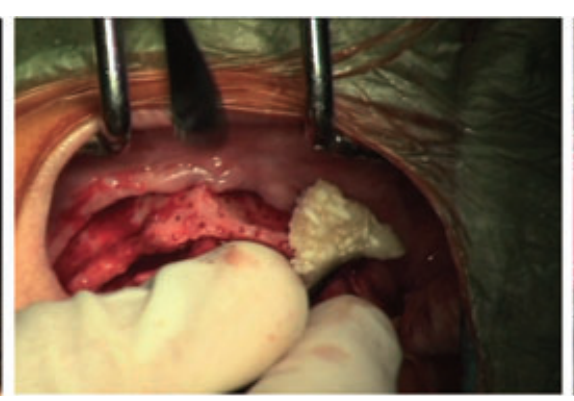

C

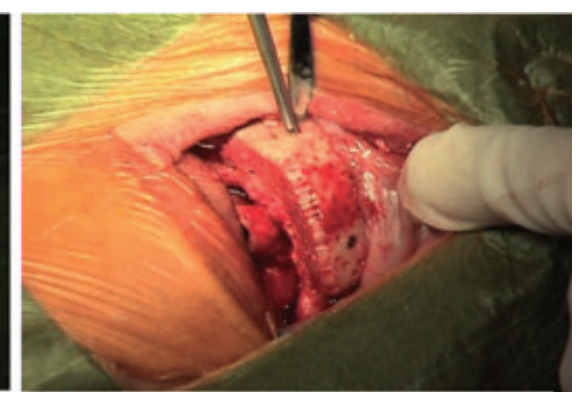

FIG. 1. (A) Syringe where the scaffold was placed and the BMA was spilled to absorb the bone marrow cells. (B,C) Clinical procedure showing the fixation of bone graft in the surgical site. 
sion in falcon tube $(15 \mathrm{~mL})$ was washed twice in rinse buffer (20 mM Tris-HCl, pH 7.4, $0.15 \mathrm{M} \mathrm{NaCl}$, $0.05 \%$ Tween-20) and permeabilized with $0.1 \%$ Triton X-100. After blocking with 5\% bovine serum albumin $(30 \mathrm{~min})$, the cells were incubated with diluted $(1: 100)$ primary antibodies for $1 \mathrm{~h}$ at room temperature. After washing three times in rinse buffer, fluorescein isothiocyanate-labeled secondary antihuman mouse antibody was added for $30 \mathrm{~min}$ at a $1: 1000$ dilution. Each protocol step was followed by brief centrifugation. Then, the cells were placed onto microscope slides pretreated with polylysine (Sigma) and were mounted in Vectashield mounting medium (Invitrogen) with 4',6-Diamidino-2-phenylindol (DAPI) (DNA binding dye). Analysis was performed with digital images that were acquired with a cooled highsensitivity VarioCam CCD camera (model VC44, PCO Computer Optics, Kelheim, Germany) and processed with ISIS software (MetaSystem, Belmont, MA, USA).

- Scanning electron microscopy: The BS with and without adhered cells were not used in the surgery. Those cells were immersed in modified Karnovsky solution containing $2.5 \%$ glutaraldehyde, $2 \%$ paraformaldehyde in $0.1 \mathrm{M}$ sodium phosphate solution ( $\mathrm{pH} \mathrm{7.4)}$ for $24 \mathrm{~h}$, rinsed with PBS, and postfixed in $1 \% \mathrm{OsO}_{4}$ in $0.1 \mathrm{M}$ sodium phosphate buffer $(\mathrm{pH} 7.4)$ for $2 \mathrm{~h}$ at $4{ }^{\circ} \mathrm{C}$ (all reagents from Sigma). Then, they were dehydrated through graded ethanol series and critical-point dried. The specimens were mounted on metal stubs, coated with gold, and observed by scanning electron microscopy (JEOL JSM-6100, Tokyo, Japan).

\section{Clinical procedures}

The clinical protocol comprised 32 patients who were submitted to sizable bone graft and placement of implants. There were 23 women between 45 and 83 years old and nine men between 58 and 75 years old. The middle age of patients was 65 years old. The surgical areas were the anterior maxilla and the posterior maxilla.

In the anterior maxilla, particulated excess of bone graft, PRP, previously obtained autogenous thrombin, and $\mathrm{CaCl}_{2}$ were mixed until a consistency of gel was achieved. This mixture was used as bedding for the bone graft.

The patients were anesthetized and an incision over the remaining alveolar ridge was performed. The bone graft was placed in position, adapted, and the residual bone was perforated to achieve a better vascular supply. The fixation of the bone graft was performed using screws and the PRP and particulate bone mixture was used to achieve a better adaptation of the graft (Fig. 1B,C).

In the posterior maxilla, alveolar ridge augmentation and sinus lifting were performed using sizable bone grafts and PRP; previously obtained autogenous thrombin and $\mathrm{CaCl}_{2}$ were mixed until a consistency of gel was achieved.

Radiographs and computed tomography (CT) sections were taken before the surgery and 8 months after the surgery. At this moment, bone biopsies were performed and implants were placed in the graft. The long-term success of the procedure was confirmed through the follow-up of the patients' implants and prosthesis.

\section{RESULTS}

\section{Characterization of cells from the BMA adhered to scaffold}

In the present study, MNCs were used together with $\mathrm{BS}$ to improve the bone grafts. For this purpose, BMA was obtained and MNCs were allowed to adhere onto the scaffold, which was later placed in the surgical area. However, it is not clear which cells from MNC fraction would mainly contribute to bone formation on the surgical area. To answer this question, small pieces of scaffold which were not used in grafts were cultured in vitro under conditions described in Material and Methods. MNC morphology from BMA was analyzed by scanning electron microscopy before and after adhesion on BS. The scaffold architecture is shown in Fig. 2A. Immediately, after the filtration of BMA, the cells that had adhered to the scaffold showed round shape morphology typical of hematopoietic cells (Fig. 2B). After $24 \mathrm{~h}$ of MNC culturing on BS in vitro, the adherent cells of fibroblast-like morphology can be observed (Fig. 2C). The presence of $\mathrm{MNC}$ on BS was also confirmed by eosin staining (Fig. 3A).

Fibroblast-like morphology of adherent cells after culturing in vitro on BS suggested their MSC origin. To verify this hypothesis, little pieces of BS (which were not used in the surgery) were collected from the culture medium and the cells were harvested from the BS. The cell suspension was obtained and immunostained with antibodies against human MSC surface antigens $\mathrm{SH}-2, \mathrm{SH}-3$, and $\mathrm{SH}-4$. Figure 3 shows that some cells were positive for $\mathrm{SH} 2$ (Fig. 3B), SH3 (Fig. 3C), and SH4 (Fig. 3D), and negative for $\mathrm{CD} 45$, which is specific for the cells of hematopoietic origin (data not shown). However, the cells which were negative for MSC markers showed positive reaction with $\mathrm{CD} 45$ indicating their hematopoietic origin (data not shown). The cells, which reacted posi- 
A

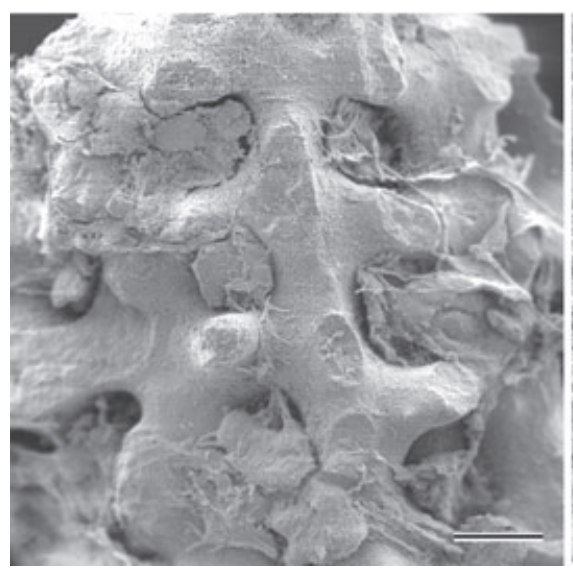

B

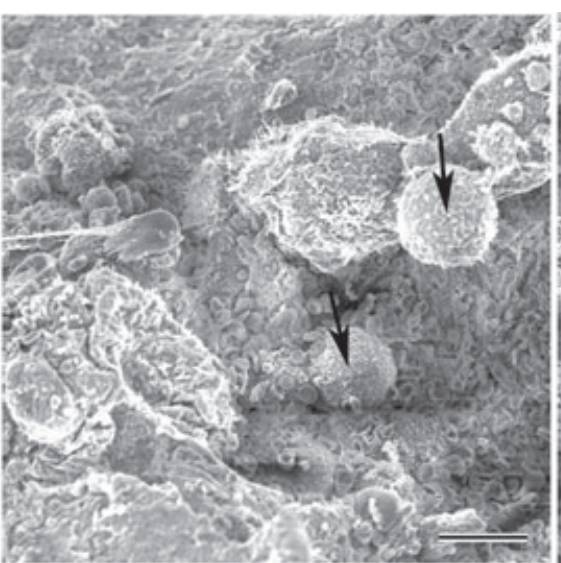

C

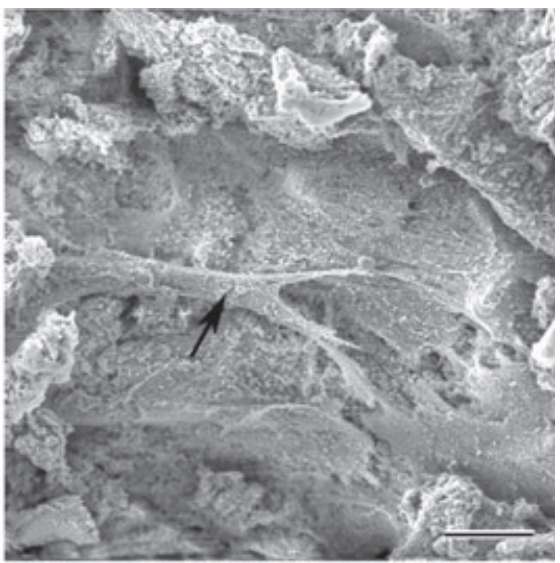

FIG. 2. Scanning electron microscopy analysis of MNC. (A) Morphological aspect of BS. Bar $=45 \mu \mathrm{m}$. (B) Cells showing round cell morphology (arrows) adherent on scaffold before culture in vitro. Bar $=25 \mu \mathrm{m}$. (C) Fibroblast-like cells observed on the scaffold $24 \mathrm{~h}$ after in vitro culture. Bar $=25 \mu \mathrm{m}$.

tively with MSC markers showed cluster formation (Fig. 3B-D), which is indicative of stem cells proliferation in culture.

\section{Clinical results}

Out of 32 bone grafts, 30 were successful (94.7\%) which means that they were osseointegrated and the amount of bone is now enough for implant placement. One of them failed due to infection in the maxillary sinus area and, in another patient, the bone graft in the anterior area was not integrated to the remaining cortical bone.

The radiographs and $\mathrm{CT}$ taken before the surgery (Fig. 4A,C) and 8 months after the surgery showed that the scaffolds were well integrated and adapted to the cortical bone (Fig. 4B,D). In all cases, the amount of bone gained in height and width was sufficient for implant placement using sequence of trephine bars. In the anterior maxilla, the width was at least $6 \mathrm{~mm}$ and in height $10 \mathrm{~mm}$ with peaks of 14 and $10 \mathrm{~mm}$, respectively. In the posterior maxilla, the height was at least $9 \mathrm{~mm}$ but the majority was over $15 \mathrm{~mm}$. In the posterior maxilla, the width measures were not significantly modified with increases of $1 \mathrm{~mm}$ or no modification.

The histological analysis of the bone biopsies, 8 months after the surgery, showed lines of bone formation and the presence of osteoblasts around the bone trabecula (Fig. 4E). All implants were in function and with almost no bone loss around them after 2 and 4 years.

\section{DISCUSSION}

The utilization of dental implants sometimes requires previous increase in the amount of bone available in the region that they must be placed. To overcome this obstacle, autologous bone grafts can
A

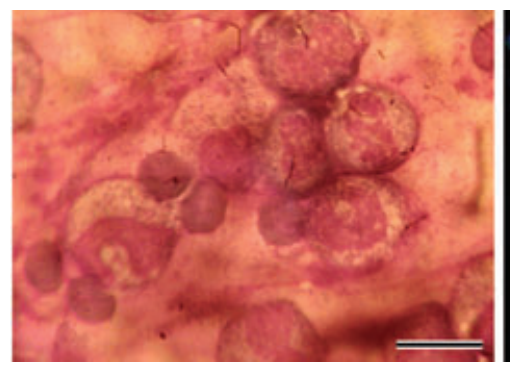

B

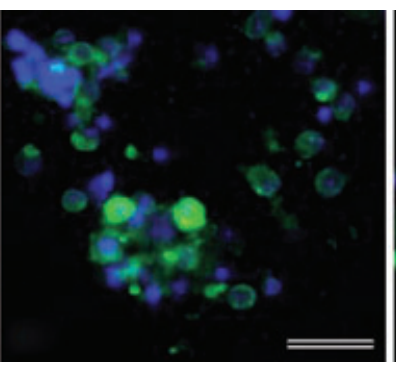

C

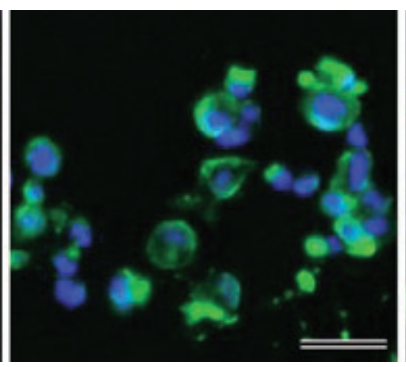

D

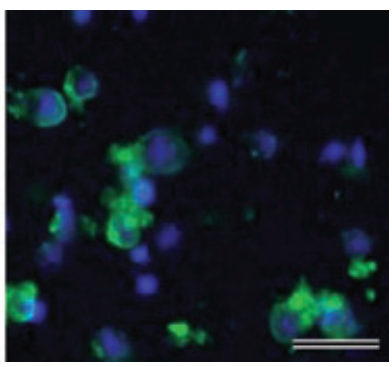

FIG. 3. Histological and immunofluorescence analysis of MNCs. (A) Eosin staining of MNCs before adhesion on BS. Bar $=25 \mu \mathrm{m}$. (B-D) MNCs harvested $24 \mathrm{~h}$ after in vitro culture on BS showing positive immunostaining (green) to MSC specific markers: $\mathrm{B}=\mathrm{SH} 2$ $($ Bar $=90 \mu \mathrm{m}), \mathrm{C}=\mathrm{SH} 3(\mathrm{Bar}=60 \mu \mathrm{m})$, and $\mathrm{D}=\mathrm{SH} 4(\mathrm{Bar}=60 \mu \mathrm{m})$. Nucleolus stained with DAPI (blue). Unlabeled cells show DAPI staining only. 

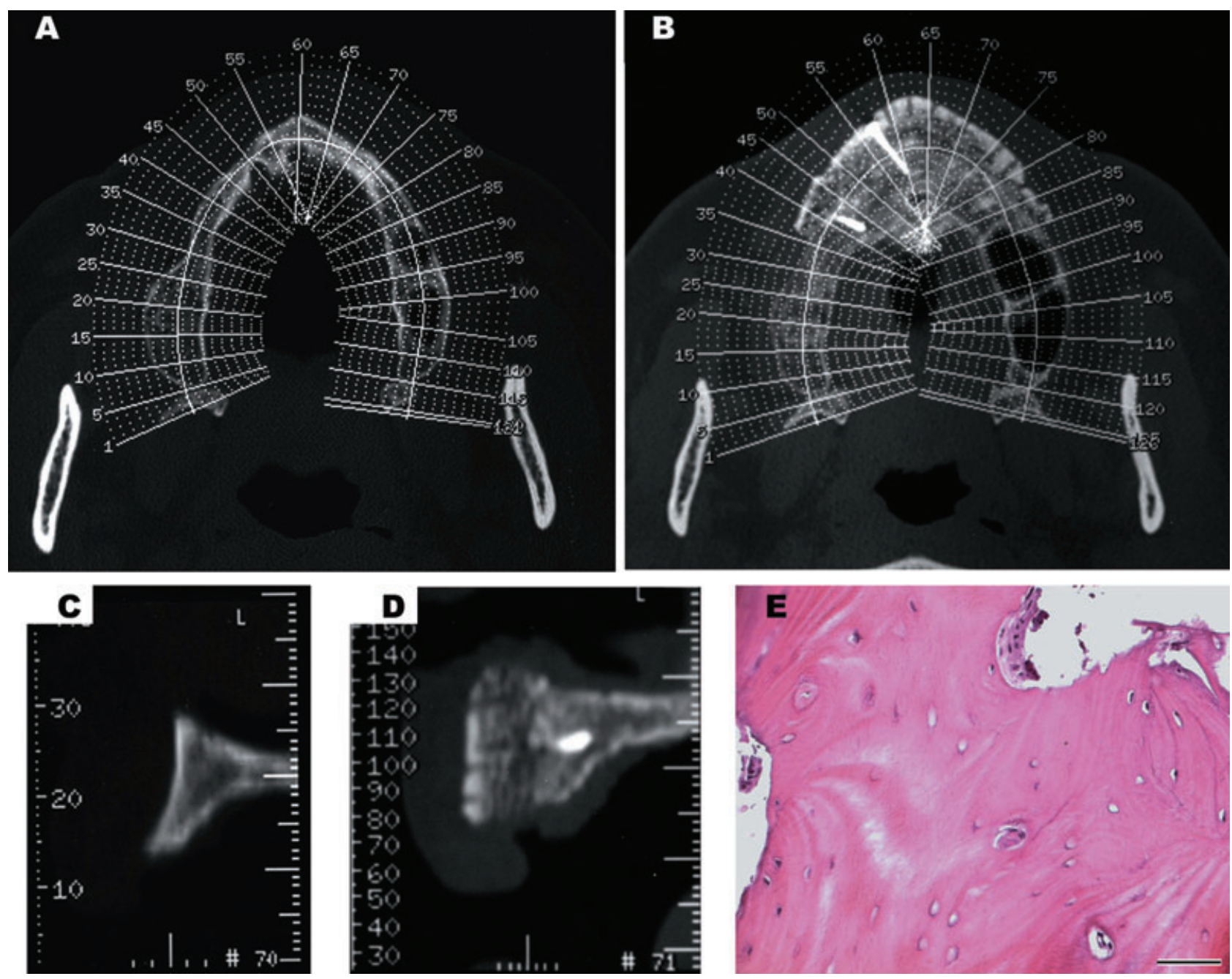

FIG. 4. CT of the surgical area before surgery $(A, C)$ and 8 months after surgery (B,D). The gain of bone and its adaptation to the remaining bone. (E) Lines of bone formation and the presence of osteoblasts around the bone trabecula. Bar $=100 \mu \mathrm{m}$.

be used although this could face serious problems concerning the donor sites' morbidity. For that reason, the nonautologous grafts are a good option.

More recently, the utilization of allogenous grafts derived from trustworthy bone banks was described, but the osseointegration of these grafts was closely related to the vascular supply of the region mainly concerning the communication with the cancellous bone which will present MCS that can differentiate in osteoblasts (16).

In addition to that, according to some researchers (17-19), the use of PRP offers many advantages in clinical dentistry. It decreases bleeding, promotes rapid soft-tissue healing and vascularization, and improves graft incorporation and bone regeneration due to its contents like platelet-derived growth factor, transforming growth factor, vascular endothelial growth factor, and insulin-like growth factor.
These factors are important for the stimulation of mitogenesis of MSC, angiogenesis, proliferation, differentiation of osteoblast precursors, and deposition of collagen matrix (20).

However, there are controversies with respect to the regenerative capacity of PRP and its use in bone grafts. Thus, other researchers have concluded that there is little scientific evidence about the benefit of PRP in maxillofacial skeletal reconstructive and preprosthetic surgery $(5,21,22)$. Sanchez et al. (21) did not demonstrate a significant improvement in bone mineral density or bone mineral content when PRP was combined with xenogeneic demineralized freezedried bone graft, and Jakse et al. (22) evaluated the regenerative capacity of PRP in a sinus autograft study on sheep, which revealed to have quite low potency. We believe that PRP plays an important role in the process of bone regeneration and the existing 
controversies can be the result of variation of growth factors content in PRP observed between the individuals and species (23). In the future, to overcome this problem more precise measures of growth factor content in each experiment will be necessary.

The use of PRP mixed with bone graft released growth factors from platelet rupture and it seems that cicatrization was improved. Then, in order to take advantage of growth factors and the ability that stem cells possess such as to originate osteoblasts, the scaffold was supplemented by the total BMA either from the iliac crest or from the sternum.

In our study, the patients received PRP and MNC from the BMA. Recently, Yamada et al. (14) made a clinical protocol using in vitro culture of MSC in the medium of animal origin, but considering the number of patients involved and the time of the follow-up of our research, this was the only possibility of MSC to be used in clinical protocol that avoids the ethical problem of in vitro culture of MSC.

Besides, we used MNC cell fraction without any preselection; our patients showed good clinical results, especially concerning the amount of bone and its characteristics. The gain of bone in the posterior maxilla in width was not significant, but the measures were over $6 \mathrm{~mm}$ in the initial tomography.

Moreover, our data suggest that the adopted method was favorable to MSC adherence and proliferation on BS after in vitro culture, as shown by immunofluorescence analysis. After filtration, the BS itself transported the MSC and hematopoietic precursors to the surgical site. Considering that MSCs are immature when compared with hematopoietic precursors and possess higher potential of proliferation and bone differentiation $(7,24)$, which is also supported by PRP-derived growth factors, it is plausible to conclude that favorable process of healing observed in the patients was due to the presence of MSC in MNC fraction in bone grafts.

\section{CONCLUSIONS}

The great advantage of using MNC fraction composed by MSC and hematopoetic precursors from BMA and growth factors (PRP) is that it promotes adequate cicatrization and integration of the graft. Our data suggest that in the future we could direct the research toward obtaining an in vitro expansion of the nucleated cells in the autologous BMA, and possibly infusing them into the patient at higher concentrations.

\section{REFERENCES}

1. Bryant SR, Zarb GA. Outcomes of implant prosthodontic treatment in older adults. J Can Dent Assoc 2002;68:97-102.
2. Stanford CM, Schneider GB. Functional behaviour of bone around dental implants. Gerodontology 2004;21:71-7.

3. Yamada Y, Ueda M, Naiki T, Nagasaka T. Tissue-engineered injectable bone regeneration for osseointegrated dental implants. Clin Oral Implants Res 2004;15:589-97.

4. Ueda M, Hibi H, Baba S. A novel approach to periodontal tissue regeneration with mesenchymal stem cells and plateletrich plasma using tissue engineering technology: a clinical case report. Int J Periodontics Restorative Dent 2006;26:363-9.

5. Schilephake H. Bone growth factors in maxillofacial skeletal reconstruction. Int J Oral Maxillofac Surg 2002;31:469-84.

6. Caplan AI. Mesenchymal stem cells. J Orthop Res 1991;9:64150.

7. Caplan AI, Bruder SP. Mesenchymal stem cells: building blocks for molecular medicine in the $21^{\text {st }}$ century. Trends $\mathrm{Mol}$ Med 2001;6:259-64.

8. Yoo JU, Johnstone B. The role of osteochondral progenitor cells in fracture repair. Clin Orthop 1998;355(Suppl.):7381.

9. Ferrari G, Cusella-De Angelis G, Coletta M, et al. Muscle regeneration by bone marrow-derived myogenic progenitors. Science 1998;279:1528-30.

10. Rosocha J, Vasko G, Bacenkova D, et al. Preliminary clinical experience with the preparation and therapeutic use of autologous osteoblasts and chondrocytes. Cell Tissue Bank 2002;3: 127-32.

11. Chen Y, Teng FY, Tang BL. Coaxing bone marrow stromal mesenchymal stem cells towards neuronal differentiation: progress and uncertainties. Cell Mol Life Sci 2006;63:164957.

12. Caplan AI. Mesenchymal stem cells: cell-based reconstructive therapy in orthopedics. Tissue Eng 2005;11:1198-1211.

13. Cancedda R, Bianchi G, Derubeis A, Quarto R. Cell therapy for bone disease: a review of current status. Stem Cells 2003; 21:610-9.

14. Yamada Y, Ueda M, Hibi H, Baba S. A novel approach to periodontal tissue regeneration with mesenchymal stem cells and platelet-rich plasma using tissue engineering technology: a clinical case report. Int J Periodontics Restorative Dent 2006; 26:363-9.

15. Zangi L, Rivkin R, Kassis I, Levdansky L, Marx G, Gorodetsky R. High-yield isolation, expansion, and differentiation of rat bone marrow-derived mesenchymal stem cells with fibrin microbeads. Tissue Eng 2006;12:2343-54.

16. Warren SM, Nacamuli RK, Song HM, Longaker MT. Tissueengineered bone using mesenchymal stem cells and a biodegradable scaffold. J Craniofac Surg 2004;15:34-7.

17. Anitua E, Andia I, Ardanza B, Nurden P, Nurten AT. Autologous platelets as a source of proteins for healing and tissue regeneration. Thromb Haemost 2004;91:4-15.

18. Luboshitz J, Berger U, Yaari O, Wiessman A, Moshonov J. Platelet rich plasma (PRP) as adjuvant tool for bone augmentation. Refuat Hapeh Vehashinayim 2004;21:63-70,103.

19. Oyama T, Nishimoto S, Tsugawa T, Shimizu F. Efficacy of platelet-rich plasma in alveolar bone grafting. J Oral Maxillofac Surg 2004;62:555-8.

20. Tozum TF, Demiralp B. Platelet-rich plasma: a promising innovation in dentistry. J Can Dent Assoc 2003;69:664.

21. Sanchez AR, Sheridan PJ, Eckert SE, Weaver AL. Regenerative potential of platelet-rich plasma added to xenogenic bone grafts in peri-implant defects: a histomorphometric analysis in dogs. J Periodontol 2005;76:1637-44.

22. Jakse N, Tangl S, Gilli R, et al. Influence of PRP on autogenous sinus grafts. An experimental study on sheep. Clin Oral Implants Res 2003;14:578-83.

23. Dolder JV, Mooren R, Vloon AP, Stoelinga PJ, Jansen JA. Platelet-rich plasma: quantification of growth factor levels and the effect on growth and differentiation of rat bone marrow cells. Tissue Eng 2006;12:3067-73.

24. Muschler GF, Nakamoto C, Griffith LG. Engineering principles of clinical cell-based tissue engineering. J Bone Joint Surg Am 2004;86:1541-58. 